\title{
Dielectric Properties of Liquid Crystalline Isothiocyanato-tolane Derivatives with Fluorine Atom at Various Lateral Positions
}

\author{
J. CzuB ${ }^{a}$, S. Urban ${ }^{a}$, R. DA̧Browski ${ }^{b}$ And B. Gestblom ${ }^{c}$ \\ ${ }^{a}$ Institute of Physics, Jagiellonian University \\ Reymonta 4, 30-059 Kraków, Poland \\ ${ }^{b}$ Institute of Chemistry, Military University of Technology \\ Kaliskiego 2, 00-908 Warsaw, Poland \\ ${ }^{c}$ Institute of Physics, Uppsala University, Uppsala, Sweden
}

(Received January 12, 2005)

\begin{abstract}
Dielectric studies were performed for several isothiocyanato-tolane compounds having the $\mathrm{F}$ atom attached to the benzene rings at different lateral positions and the alkoxy or alkyl chain at the $p$-position. They form the nematic phase in broad temperature intervals. The static as well as dynamic properties of the compounds in the nematic and isotropic phases were studied. Tensor permittivity components: $\varepsilon_{\|}, \varepsilon_{\perp}, \Delta \varepsilon$, as functions of temperature were measured. The complex dielectric permittivity, $\varepsilon^{*}(f)=\varepsilon^{\prime}(f)-\mathrm{i} \varepsilon^{\prime \prime}(f)$, was measured in the frequency range of $1 \mathrm{kHz}-$ $3 \mathrm{GHz}$ from which the relaxation times $\tau_{\|}$and $\tau_{\perp}$ were calculated. The order parameter $S(T)$ was determined from the dielectric data. It was found that the close vicinity of the $\mathrm{F}$ atom and the alkoxy group results in blocking of the internal rotation of the wing around the O-phenyl bond.
\end{abstract}

PACS numbers: $64.70 . \mathrm{Md}, 77.22 . \mathrm{Gm}, 77.84 . \mathrm{Nh}$

\section{Introduction}

The application of liquid crystals (LCs) to design displays with sufficiently good time responses stimulates chemists to search for new classes of mesogenic substances. Such synthetic works are systematically carried out at the Institute of Chemistry of the Military Technical University in Warsaw (e.g. [1-3]). The knowledge of the dielectric properties of LC substances (tensor permittivity com- 
ponents $\varepsilon_{\|}, \varepsilon_{\perp}$, dielectric anisotropy $\Delta \varepsilon=\varepsilon_{\|}-\varepsilon_{\perp}$, relaxation times $\tau_{\|}$and $\tau_{\perp}$ ) are essential for that purpose. Recently, several new series of compounds based on the tolane core were synthesized [1]. Parts of them have the isothiocyanato, -NCS, terminal group at the one side and the alkyl or alkyloxy group at the other side. They exhibit the crystal-like smectic phases (Sm E and/or Sm K) [1-3]. Particularly those seem to be interesting which in addition have the $\mathrm{F}$ atom at different lateral positions. They form the nematic phase in broad temperature ranges and have big birefringence. The present paper reports on the dielectric studies of this class of substances. Both static and dynamic properties of the compounds were studied.

\section{Experimental}

The substances studied are presented in Table I. All of them possess the same central tolane core. The first three compounds have the $\mathrm{F}$ atom attached to the phenyl-alkoxy moiety and differ by the length of the alkoxy chain. The compound with $\mathrm{F}$ replaced by $\mathrm{Cl}$, as well as the 1:1 mixture of both, belongs to the same group. The other three compounds have the $\mathrm{F}$ atom attached to the isothiocyanato-phenyl moiety at the meta- or orto-positions. Such shifting of the $\mathrm{F}$ atom within the molecular core leads to different dipole structures which should be reflected in the dielectric properties studied.

The dielectric relaxation spectra, $\varepsilon^{*}(f)=\varepsilon^{\prime}(f)-\mathrm{i} \varepsilon^{\prime \prime}(f)$, were recorded with the aid of two set-ups: an impedance analyzer (Agilent 4194A, $1 \mathrm{kHz}-10 \mathrm{MHz}$ ) and a time domain spectrometer (TDS, $10 \mathrm{MHz}-3 \mathrm{GHz}$ ). The samples in the nematic phase were oriented by a magnetic field of $c a .0 .8 \mathrm{~T}$. Two experimental geometries were applied: $\boldsymbol{E} \| \boldsymbol{B}$ and $\boldsymbol{E} \perp \boldsymbol{B}$, enabling the measurement of the $\varepsilon_{\|}$and $\varepsilon_{\perp}$ permittivity tensor components, respectively. The temperature was stabilized within $\pm 0.2 \mathrm{~K}$.

\section{Results}

The tensor permittivity components measured for the members of the $n$ OFTOL homologous series and for the other substances are presented in Figs. 1 and 2, respectively. As is seen in the figures the values of the permittivity components as well as the values of the dielectric anisotropy in the $\mathrm{N}$ phase depend on the alkoxy chain length and on the position of the $\mathrm{F}$ atom with respect to the long molecular axes.

Typical relaxation spectra obtained for the isotropic phase with the aid of TDS are presented in Fig. 3. They clearly show two well separated relaxation domains. These should be ascribed to the two main rotational movements of the molecules - around the short and the long molecular axes — in conjunction with the non-zero longitudinal and transverse components of the dipole moment. The relaxation spectra can be described by a superposition of two relaxation processes 
TABLE I

Substances studied with the acronyms used and temperatures of phase transitions observed in dielectric studies during the cooling runs. Temperatures of the melting (m.p.) are also given.

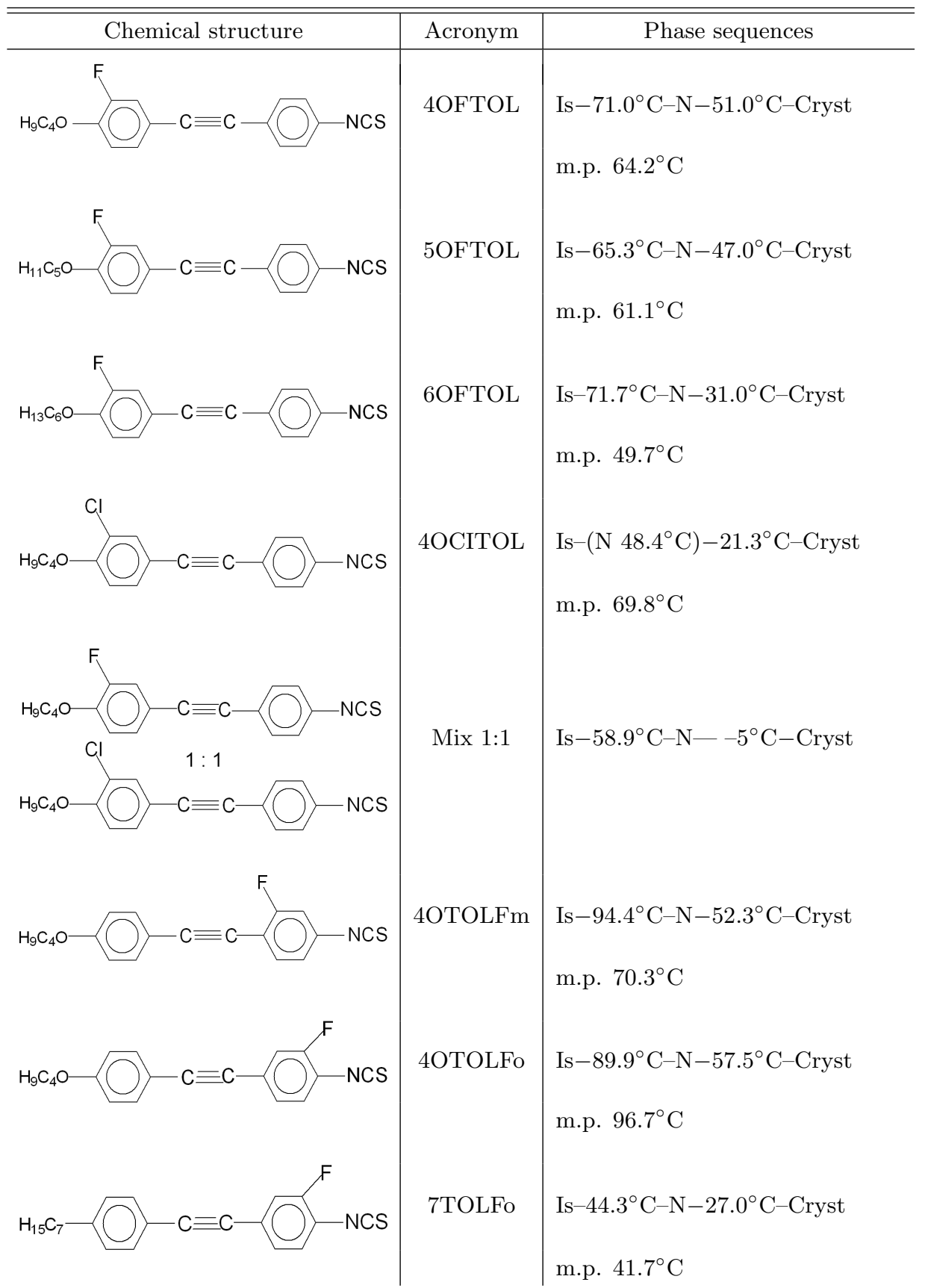



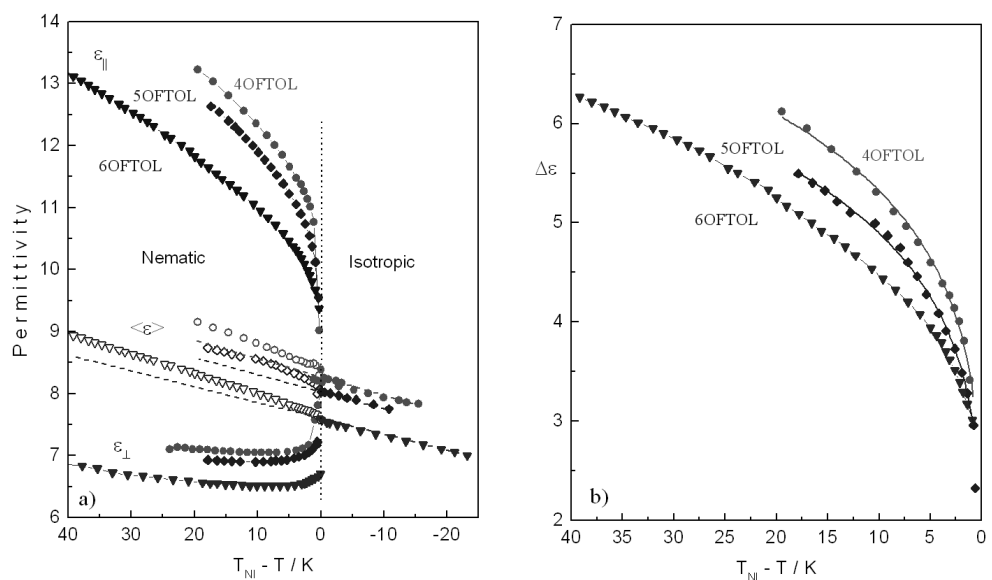

Fig. 1. (a) Tensor permittivity components versus the reduced temperature obtained for three $n$ OFTOL substances having different lengths of the alkoxy group; in all cases the mean permittivity in the $\mathrm{N}$ phase is slightly larger than the value extrapolated from the isotropic phase. (b) Dielectric anisotropy for these substances.
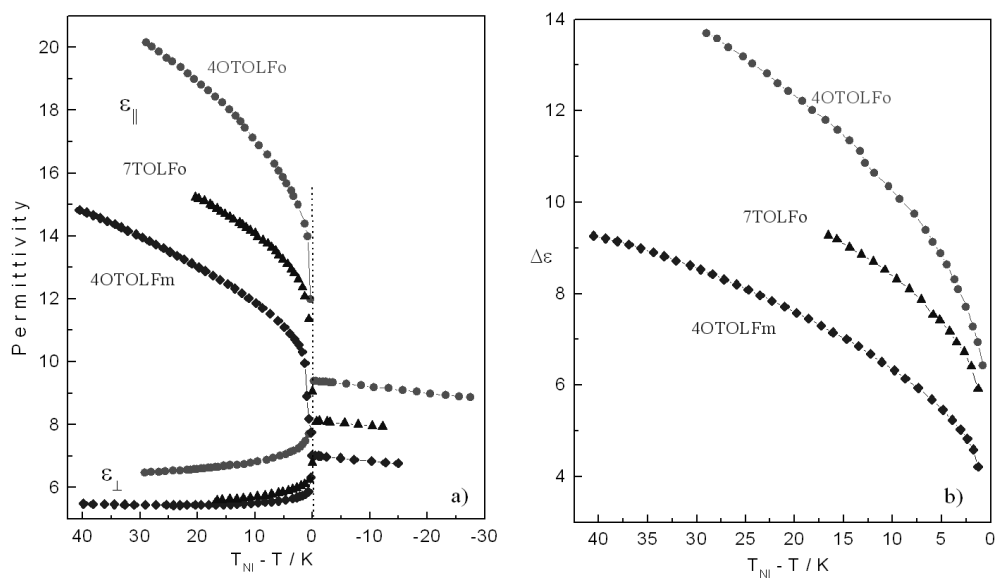

Fig. 2. (a) Tensor permittivity components versus the reduced temperature obtained for three substances having the $\mathrm{F}$ atom at different positions. (b) Dielectric anisotropy for these substances.

connected with the molecular reorientations around the short and long axes:

$$
\varepsilon^{*}-\varepsilon_{\infty}=\frac{\delta_{1}}{\left(1+\mathrm{i} \omega \tau_{1}\right)^{1-\alpha_{1}}}+\frac{\delta_{2}}{\left(1+\mathrm{i} \omega \tau_{2}\right)^{1-\alpha_{2}}},
$$

where $\delta_{1}=\epsilon_{\mathrm{s}}-\varepsilon_{2}, \delta_{2}=\varepsilon_{2}-\varepsilon_{\infty}$, are the strengths (increments) of both relaxation processes, $\varepsilon_{\mathrm{s}}$ and $\varepsilon_{\infty}$ are the static and high frequency permittivity, respectively, and $\alpha$ characterizes a distribution of the relaxation times. The dashed lines in 


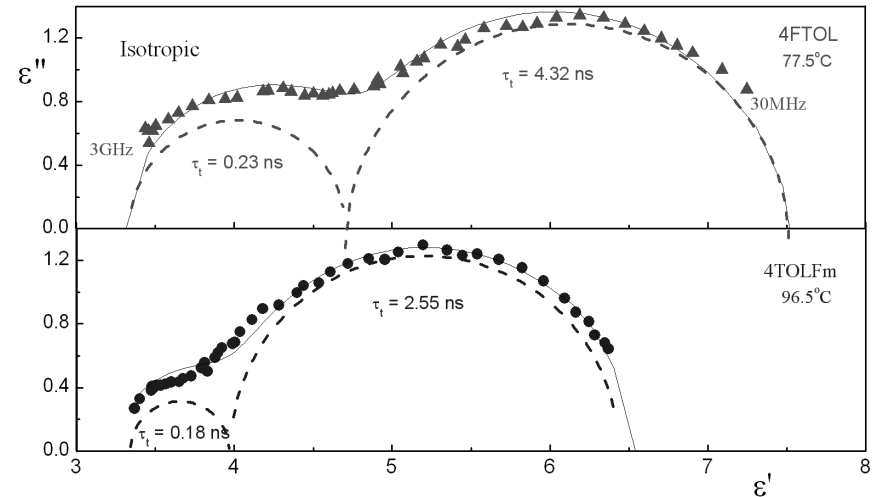

Fig. 3. The Cole-Cole plots for two substances in the isotropic phase. The solid line is the fit of Eq. (1) to the spectra, and the dashed lines show the both contributing relaxation processes with relaxation times indicated.
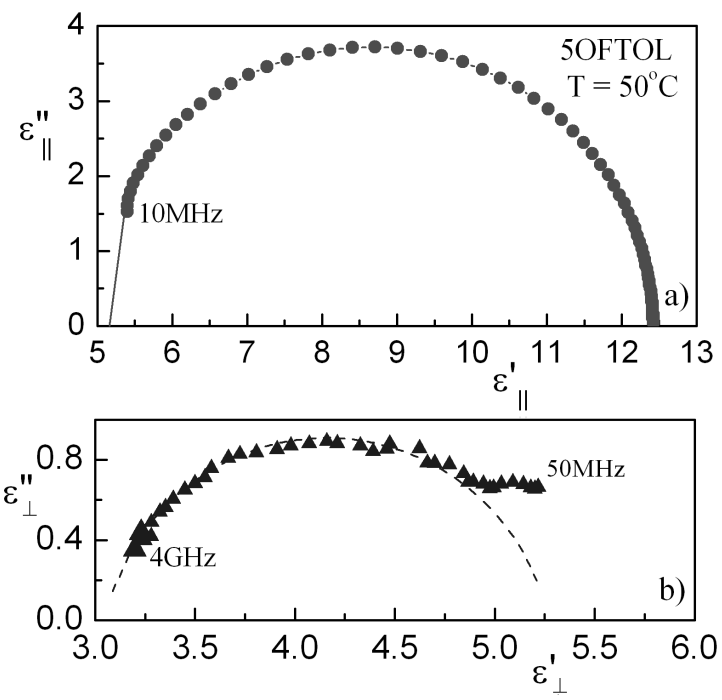

Fig. 4. The Cole-Cole plots for 5OFTOL in the $\mathrm{N}$ phase for samples oriented parallel (a) and perpendicular (b) to the measuring field. In the case of (b) the l.f. process influences the spectrum due to imperfect alignment of the sample.

Fig. 3 show the decomposition into two relaxation processes resulting from a fit of Eq. (1) to the spectra. The low frequency process can be well treated as a Debye process because the $\alpha_{1}$ parameter was close to zero, whereas the high frequency process shows a small distribution of the relaxation times $\left(\alpha_{2} \cong 0.05 \div 0.1\right)$. In the nematic phase the low frequency process $(\boldsymbol{E} \| \boldsymbol{B}$ case $)$ is purely Debye-type for all substances studied (Fig. 4a). In the case of $\boldsymbol{E} \perp \boldsymbol{B}$ alignment (Fig. 4b), besides the high frequency process connected with the fast reorientations of molecules around 
the long axes, the low frequency process is also seen due to imperfect alignment of the sample.

Figure 5 shows the Arrhenius plots for the $n$ OFTOL substances. The low frequency (l.f.) relaxation time exhibits a jump at the clearing point, whereas the high frequency (h.f.) relaxation time passes smoothly through the transition point. The retardation factor, $g_{\|}=\tau_{\|} / \tau_{\text {l.f. }}$, equals $3.6,3.8$, and 5.3 for $n=4,5$, and 6 , respectively.

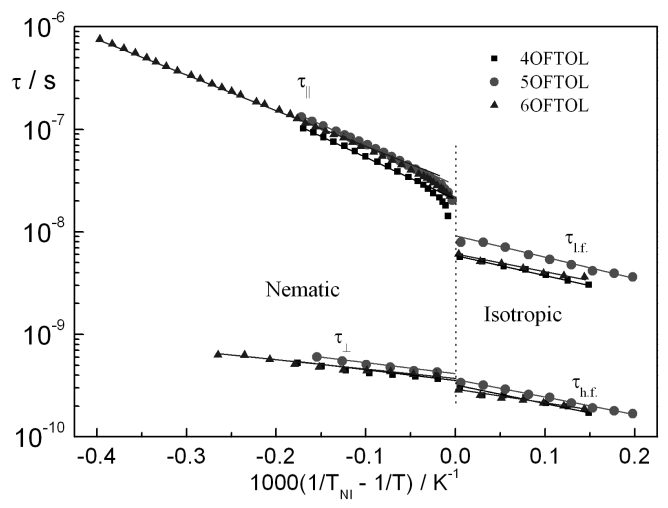

Fig. 5. Arrhenius plot from the relaxation times determined for $n$ OFTOL compounds in the isotropic and nematic phases. In order to avoid shifts due to different clearing temperatures in the abscissa the term $\left(1 / T_{\mathrm{NI}}-1 / T\right)$ has been used against $1 / T$.

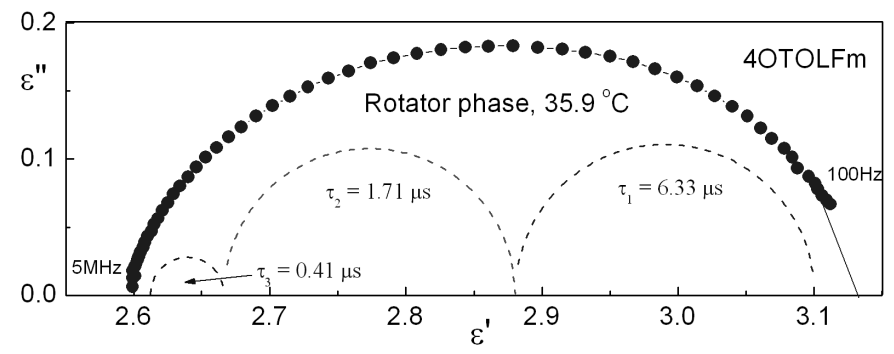

Fig. 6. The Cole-Cole plot for 4OTOLFm in the solid rotator phase. The solid line is the fit of Eq. (1) to the spectra, and the dashed lines correspond to three Debye-type relaxation processes. The calculated relaxation times are presented in Fig. 7.

In the case of 4OTOLFm the nematic phase transformed reversibly to a solid rotator phase which persisted down to room temperature and could be kept in this state for several days (however, the virgin sample was a normal solid at room temperature). Figure 6 presents the broad relaxation spectrum measured in this state indicating the presence of several processes. Three relaxation domains can be consistently fitted to the spectra in broad temperature intervals. This 
seems to indicate that one deals with a solid-like phase in which the molecules as a whole and their polar parts perform independent rotation motions around the long axis. However, due to steric hindrances the relaxation times as well as the activation barriers are of the same order of magnitudes as those characterizing the molecular rotation around the short axis in the $\mathrm{N}$ phase (Fig. 7, Table II). Looking at the chemical structure of the molecule (Table I) and taking into account the observed dielectric increments one can suppose that $\tau_{1}, \tau_{2}$, and $\tau_{3}$ correspond to the rotation of the whole molecule, the rotation of the moiety with the $\mathrm{F}$ atom and the rotation of the alkoxy group, respectively.

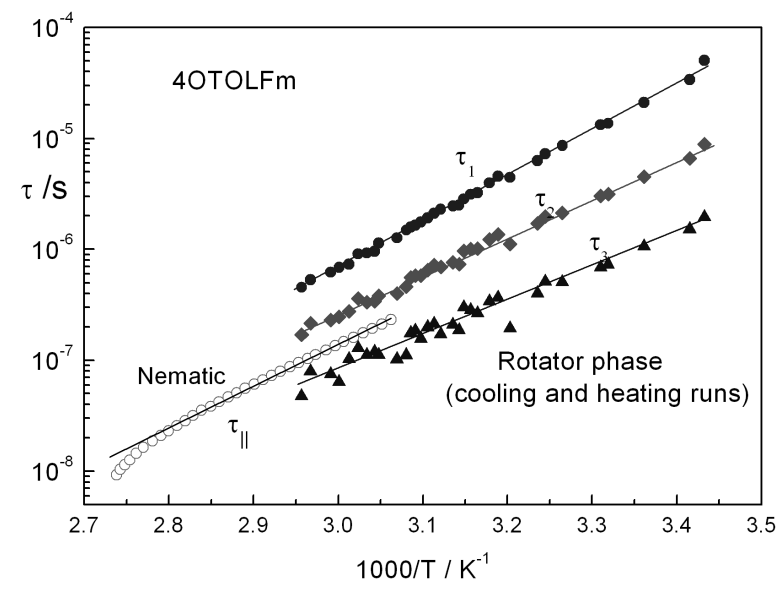

Fig. 7. Arrhenius plots from the relaxation times determined for 4OTOLFm in the nematic and solid rotator phases.

TABLE II

Activation enthalpy values (in $\mathrm{kJ} / \mathrm{mol}$ ) calculated with the Arrhenius equation, $\tau=\tau_{0} \exp (\Delta H / R T)(R-$ gas constant) for relaxation processes observed in different substances.

\begin{tabular}{c|c|c|c|c|c|c|c}
\hline \hline Phase & \multicolumn{2}{|c|}{ Isotropic } & \multicolumn{2}{|c|}{ Nematic } & \multicolumn{3}{c}{ Solid rotator } \\
\hline Substance & l.f. & h.f. & l.f. & h.f. & l.f. & m.f. & h.f. \\
\hline 4OFTOL & 36.4 & 27.7 & 79.3 & 29.3 & & & \\
5OFTOL & 37.5 & 24.3 & 79.1 & 32.9 & & & \\
6OFTOL & 30.6 & 23.4 & 72.9 & 20.3 & & & \\
Mix 1:1 & & & 80 & & & & \\
4OTOLFm & & & 71.5 & & 79.0 & 66.3 & 57.0 \\
4OTOLFo & & & 82.4 & & & & \\
7TOLFo & 33.8 & 24.8 & 62.9 & & & &
\end{tabular}



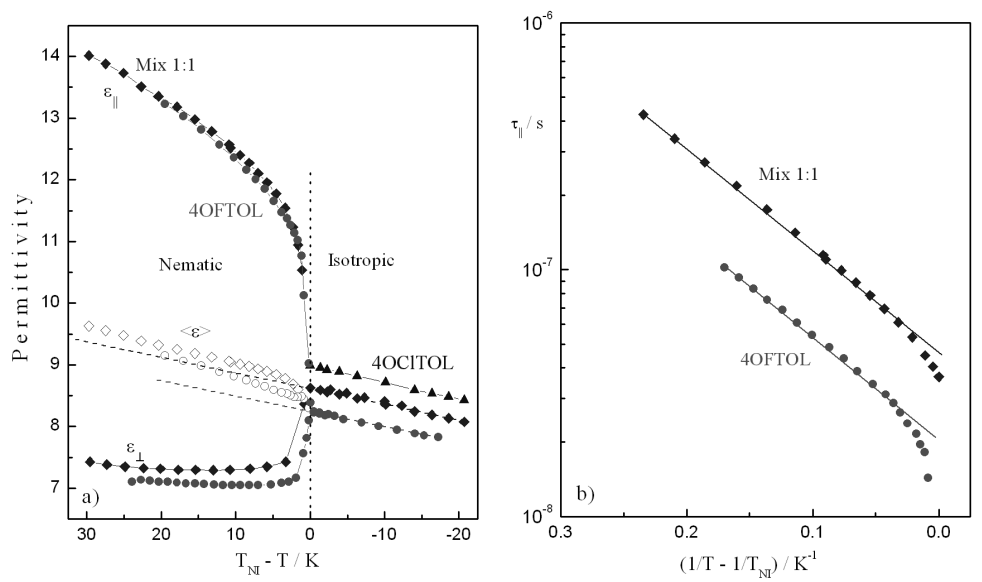

Fig. 8. (a) The permittivity components in the nematic and isotropic phases of the pure 4OFTOL and 4OClTOL and their 1:1 mixture. (b) The l.f. relaxation time in the nematic phase of 4OFTOL and its 1:1 mixture with 4OCITOL.

The exchange of the fluorine atom for a chlorine atom in the 4OFTOL (Table I) results in disappearing of the $\mathrm{N}$ phase (in fact, the 4 OClTOL substance exhibits a monotropic $\mathrm{N}$ phase which, however, could not be observed in the dielectric experiment). The 1:1 molar mixture has again the enantiotropic $\mathrm{N}$ phase in a broad temperature interval. The dielectric properties of pure substances and the mixture are compared in Fig. 8a. The particular permittivity components differ only slightly. The same concerns the activation enthalpy for the l.f. process (Table II), although the relaxation time of the mixture is shifted to longer values due to a higher mass of the chlorine component (Fig. 8b). However, the relaxation spectra of the mixture do not show a noticeable distribution of the relaxation times.

\section{Discussion}

From the point of view of the dielectric spectroscopy it is important to note that all the molecules studied possess three dipole groups: $\varnothing-\mathrm{NCS}(\mu=3.34 \mathrm{D}$ along the $p$-axis), $\varnothing-\mathrm{F}$ ( $\mu=1.47 \mathrm{D}$ inclined by $120^{\circ}$ from the $p$-axis), $\varnothing$ stands for the benzene ring, and $-\mathrm{OC}_{n} \mathrm{H}_{2 n+1}\left(\mu=1.22 \mathrm{D}\right.$ inclined by $\left.72^{\circ}\right)$, compare Fig. $9[4,5]$.

Using the group dipole moments $(\mathrm{GDM})$ method $[4,5]$ one can calculate the values of the transverse $\mu_{\mathrm{t}}$ and longitudinal $\mu_{1}$ components and thus the net dipole moment and the angle $\beta$ which it forms with the $p$-axis of the molecule. (It should be mentioned that this axis might differ from the lowest inertia moment axis treated as the long axis of the molecule due to conformation motions of the alkoxy group.) The obtained values are gathered in Table III (the second and third columns). 


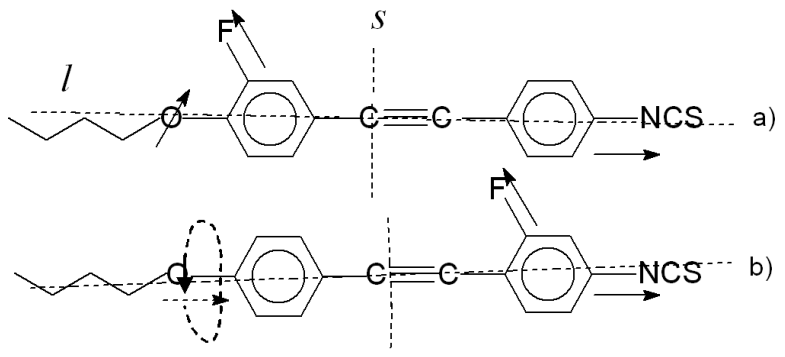

Fig. 9. Chemical structures of 4OFTOL (a) and 4OTOLFm (b) compounds. The dashed lines indicate the long $(l)$ and the short $(s)$ molecular axes. In the case of 4OFTOL the interaction of the $\mathrm{F}$ atom with the alkoxy group leads to blocking the wing rotation around the para axis, whereas for 4OTOLFm due to fast rotation of the alkoxy group the perpendicular component of the dipole moment becomes averaged to zero.

\section{TABLE III}

The estimated dipole moment and the values of the angle $\beta$ according to the GDM calculations and the Onsager equation applied to the isotropic liquid.

\begin{tabular}{c|c|c|c}
\hline \hline Substance & $\mu_{\text {est }}[\mathrm{D}]$ & $\beta\left[^{\circ}\right]$ (from GDM) & $\beta\left[^{\circ}\right]$ (from Onsager) \\
\hline 4OFTOL & 3.84 & 39.2 & 41.6 \\
5OFTOL & 3.84 & 39.2 & 42.8 \\
6OFTOL & 3.84 & 39.2 & 43.9 \\
4OClTOL & 3.88 & 40.8 & 41.3 \\
4OTOLFm & 3.20 & 23.1 & 27.4 \\
4OTOLFo & 4.70 & 26.5 & - \\
7TOLFo & 4.27 & 17.4 & 24.0
\end{tabular}

On the other hand, the angle $\beta$ can be estimated from the dielectric data for the isotropic phase. Figure 3 presents the relaxation spectra in the form of the Cole-Cole plots for 4OFTOL and 4OTOLFm which would seem to have close values of the longitudinal $\left(\mu_{\mathrm{l}}\right)$ and transverse $\left(\mu_{\mathrm{t}}\right)$ components of the dipole moment (compare chemical structures in Table I). One should then expect that the dielectric increments for the two main relaxation processes would be similar for both substances. As can be seen in Fig. 3 the increments for 4OTOLFm are considerably smaller in comparison with the 4OFTOL case. This indicates a non equivalent role of the $\mathrm{F}$ atom in both chemical structures. By applying the Onsager equation,

$$
\frac{\left(\varepsilon_{\mathrm{s}}-\varepsilon_{\infty}\right)\left(2 \varepsilon_{\mathrm{s}}+\varepsilon_{\infty}\right)}{\varepsilon_{\mathrm{s}}\left(\varepsilon_{\infty}+2\right)^{2}}=\frac{N_{0}}{3 \varepsilon_{0}} \frac{\mu^{2}}{3 k T},
$$

separately to the low frequency increment, governed by the longitudinal compo- 
nent $\mu_{1}$, and to the high frequency increment, governed by the transverse component $\mu_{\mathrm{t}}$, one can calculate the angle $\beta=\tan ^{-1}\left(\mu_{\mathrm{t}} / \mu_{\mathrm{l}}\right)$. The obtained values are presented in the fourth column of Table III. Rather good agreement can be noted for $n$ OFTOLs and 4OCITOL which indicates that in each case the long molecular axis is close to the symmetry axis of the molecular core. However, in the isotropic phase of 4 OTOLFm the static permittivity $\left(\varepsilon_{\mathrm{s}}=6.4\right)$ is markedly smaller than that observed for 4OFTOL $\left(\varepsilon_{\mathrm{s}}=7.6\right)-$ see Fig. 2. Moreover, the reduction of increments becomes considerably larger for the transverse than for the longitudinal components $\left(\delta_{\mathrm{t} 2} / \delta_{\mathrm{t} 1}=0.41, \delta_{12} / \delta_{11}=0.89\right.$, where 1 and 2 refers to 4 OFTOL and 4 OTOLFm, respectively). The first fact points to a decrease in the total dipole moment in the case of 4OTOLFm, the second one indicates that the larger reduction concerns the transverse component of the dipole moment. The possible explanation of that is the following. The presence of the $\mathrm{F}$ atom close to the alkoxy group in $n$ OFTOLs stops the internal rotation of the alkoxy tail around the $\varnothing-\mathrm{O}$ axis, whereas in the case of 4 OTOLFm this rotation averages the perpendicular component of the dipole moment to zero and the alkoxy group gives the contribution to the longitudinal dipole moment only. In such case $\beta(\mathrm{GDM})=23.2^{\circ}$ being in reasonable agreement with $\beta$ (Onsager $)=27.4^{\circ}$.

The order parameter $S(T)$ is essential for describing the physical properties of the nematic phase. It can be determined by measurements of all anisotropic quantities, like optical and dielectric anisotropies, magnetic susceptibility and quadrupole splitting (eg. [6-9] and references therein). Recently we have shown $[8,9]$ that for substances with a large positive dielectric anisotropy the Maier and Meier equation [10], as well as the Coffey et al. [11, 12] approach based on the retardation factor, can give valuable information on the order parameter as a function

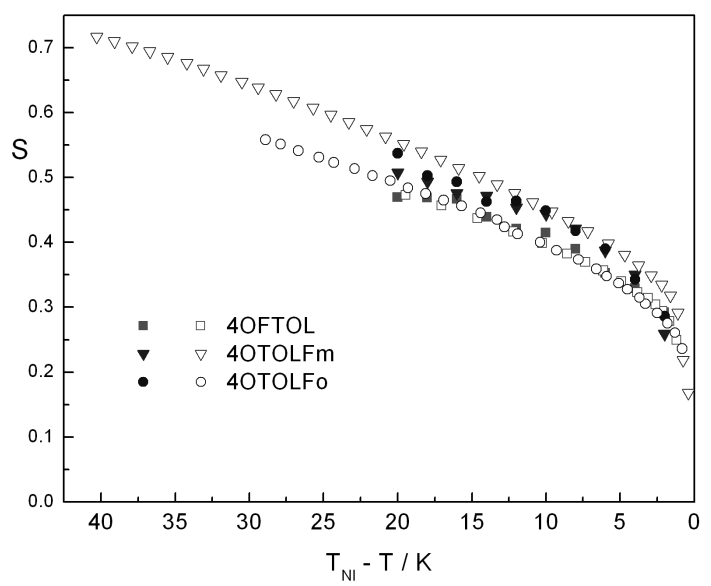

Fig. 10. Order parameter for three substances as determined from the refractive index measurements (full symbols) and from the dielectric anisotropy data (open symbols). 


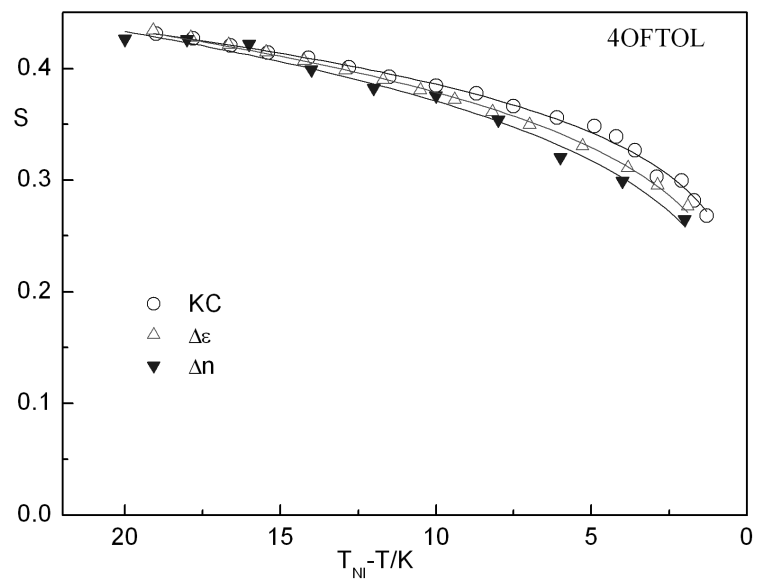

Fig. 11. Order parameter for the nematic phase of 4OFTOL determined from the optical anisotropy $(\Delta n)$, dielectric anisotropy $(\Delta \varepsilon)$, and retardation factor $[11,12](\mathrm{KC})$.

TABLE IV

The values of the parameters in the Haller formula for substances studied in the nematic phase.

\begin{tabular}{c|c|c}
\hline \hline Substance & $S_{0}$ & $\gamma$ \\
\hline 4OFTOL & 0.360 & 0.175 \\
5OFTOL & 0.361 & 0.175 \\
6OFTOL & 0.377 & 0.167 \\
4OTOLFm & 0.296 & 0.206 \\
4OTOLFo & 0.297 & 0.206 \\
7TOLFo & 0.365 & 0.175
\end{tabular}

of temperature and pressure. The mentioned procedures were now applied to the present substances. Figure 10 presents the order parameter $S(T)$ determined from the optical anisotropy measurements [13] (full symbols) and from the dielectric anisotropy (open symbols). In all cases the $S$-values were treated according to the Haller formula [14]: $S(T)=S_{0}\left(T_{\mathrm{NI}}-T\right)^{\gamma}$, with $S_{0}=\left(T_{\mathrm{NI}}\right)^{-\gamma}$, whereas $\gamma$ was determined from the fitting this formula to the experimental points. Both sets of points are in fairly good agreement. Also the data determined for the same substance with the aid of different approaches are in a good concordance - Fig. 11. Table IV contains the values of $S_{0}$ and $\gamma$ determined for particular substances studied.

\section{Summary}

Dielectric studies were performed for several isothiocyanato-tolane compounds having the $\mathrm{F}$ atom attached to the benzene rings at different lateral po- 
sitions, and the alkoxy or alkyl chain at the $p$-position. The tensor permittivity components as functions of temperature within the nematic phase were determined. The dielectric relaxation times characterizing two main rotation motions of molecules around the principal inertia axes were established for the nematic and isotropic phases. Difference in the dielectric permittivity values observed for compounds with the $\mathrm{F}$ atom attached to the alkoxy-phenyl or isothiocyanato-phenyl rings enabled us to conclude that the close vicinity of the $\mathrm{F}$ atom and the alkoxy group results in blocking of the internal rotation of the wing around the O-phenyl bond. This leads to a good concordance of the $\beta$ angle values, as determined using the group dipole moment method and that estimated from the dielectric increments measured in the isotropic phase. The dielectric data served us to determine the order parameter being in a good concordance with that calculated from the optical anisotropy.

\section{References}

[1] A. Spadło, J. Dziaduszek, W. Drzewiński, R. Dạbrowski, C.Y. Fan, S.-T. Wu, Liq. Cryst. 30, 191 (2003).

[2] R. Dąbrowski, J. Przedmojski, A. Spadło, J. Dziaduszek, M. Tykarska, Phase Trans. 77, 1103 (2004).

[3] S. Urban, J. Czub, R. Dąbrowski, H. Kresse, Liq. Cryst. 32, 119 (2005).

[4] W.I. Minkin, O.A. Osipov, Yu.A. Zhdanov, Dipole Moments in Organic Chemistry, Plenum Press, New York 1970.

[5] O. Exner, Dipole Moments in Organic Chemistry, Georg Thieme Publishers, Stuttgart 1975

[6] G.R. Luckhurst, in: The Molecular Physics of Liquid Crystals, Eds. G.R. Luckhurst, G.W. Gray, Academic Press, Oxford 1979, ch. 4.

[7] C. Zannoni, in: The Molecular Dynamics of Liquid Crystals, Eds. G.R. Luckhurst, C.A. Veracini, NATO series, Vol. 431, Kluwer Academic Publishers, Dordrecht 1994, ch. 2.

[8] S. Urban, B. Gestblom, W. Kuczyński, S. Pawlus, A. Würflinger, Phys. Chem. Chem. Phys. 5, 924 (2003).

[9] S. Urban, B. Gestblom, S. Pawlus, Z. Naturforsch. A 58, 357 (2003).

[10] W. Maier, G. Meier, Z. Naturforsch. A 16, 262, 470 (1961).

[11] W.T. Coffey, Yu.P. Kalmykov, J.P. Waldron, Liq. Cryst. 18, 677 (1995).

[12] Yu.P. Kalmykov, W.T. Coffey, Liq. Cryst. 25, 329 (1998).

[13] A. Spadło, R. Dąbrowski, J. Dziaduszek, S. Urban, E. Ścibior, S. Gauza, S.T. Wu, to be published in Appl. Opt.

[14] I. Haller, Prog. Solid State Chem. 10, 103 (1975). 